\title{
Editorial
}

\section{Focusing the Geriatric Population: Cardiovascular Diseases}

\author{
AAS Majumder, MD, FACC, FRCP \\ Professor of Cardiology, National Institute of Cardiovascular Diseases, Dhaka
}

Geriatric population i.e. those with an age of 65years or more constitute $6 \%$ population. With the advancement of medical science longevity has much increased. So proportion of geriatric population is gradually increasing day by day.

Old age is not a disease by itself though the elderly are vulnerable to long term diseases of insidious onset such as cardiovascular illness, cerebrovascular diseases, cancer, diabetes, musculoskeletal and mental illnesses. They have multiple symptoms due to decline of various body functions. ${ }^{1}$ Many of the health problems associated with aging can be prevented or delayed if people have access to suitable health care services.

Treating geriatric patients requires a different strategy and is not simple. Additional awareness and focus is needed. Common errors include causing iatrogenic problems by polypharmacy or by prescribing normal adult doses to elderly patients. ${ }^{2}$ For example, most of the elderly patients with heart failure suffer from renal impairment. In those patient use of normal dose of digoxin can produce deleterious consequences. Presence of malignancy influences the treatment plan of a patient and it is more pronounced in geriatric population.

The vast majority of outcome studies show that good geriatric care results in better quality of life, lower morbidity and lower mortality. ${ }^{3}$

Acutely decompensated heart failure has become the leading cause of hospitalization for people aged 65 or older. Heart failure is mainly a disease of the elderly with prevalence rates increasing from $0.7 \%$ in those aged $55-64$ years, to $2.7 \%$ in those aged 65 74 years and $13 \%$ in those aged $75-84$ years. The presence of heart failure in older patients results in an approximately 50\% reduction in life expectancy. The time has come to undertake active research to gain a better understanding of the basis of heart failure in the elderly and provide effective management of heart failure. ${ }^{4}$

The etiology of secondary pulmonary hypertension in geriartric population is mainly attributed to systemic hypertension, interstitial/inflammatory pulmonary disorders, systolic and diastolic dysfunction of heart. History of airway disease, pulmonary embolism, and valvular dysfunction are also important. Aggressive treatment of cardiopulmonary disorders in geriartric population can delay or even stop the development of the secondary pulmonary hypertension. ${ }^{5}$

Increases in blood pressure (BP), particularly systolic BP, was traditionally considered to be a normal or "physiologic" component of the aging process. However, it is now clear that elevated BP, particularly systolic BP, represents a pathophysiologic manifestation of altered cardiovascular physiology and structure, ultimately manifesting as increased cardiovascular morbidity and mortality (myocardial infarction, stroke and total cardiovascular death rates). More than one half of the population aged 65 or older have hypertension, defined as $\mathrm{BP} \geq 140 / 90 \mathrm{~mm} \mathrm{Hg}$. Encouraging data from clinical trials now strongly support the aggressive anti-hypertensive treatment of elderly patients. A recent meta-analysis of eight outcome trials evaluating the risks of treated and untreated isolated systolic hypertension has demonstrated a $30 \%$ reduction in combined fatal and nonfatal stroke, a $26 \%$ reduction in fatal and nonfatal cardiovascular events, and a $13 \%$ reduction in total mortality. In general, the elderly should be treated to target BP levels identical to those suggested for younger patients, although a more gradual reduction to target, perhaps with an intermediate BP goal of $<160 \mathrm{~mm} \mathrm{Hg}$, may be advisable. ${ }^{6}$

Acute coronary syndrome in the elderly is being undertreated both medically and by coronary interventions because of fear of bleeding and other 
complications. ${ }^{7}$ On the other hand, elderly patients represents a very high risk group that may benefit the most from potent antithrombotic regimens and coronary interventions that have been shown to improve clinical outcome significantly in younger patients. An invasive approach has already been shown to be cost effective than optimized medical therapy in elderly patients with chronic stable angina. ${ }^{8}$ Revascularization in patients presenting with high risk non-ST-elevation acute coronary syndrome is associated with significant benefits at 6 months, independent of age and does not increase the risk of stroke. ${ }^{9}$

Aortic stenosis is the most common form of acquired valvular heart disease in developed as well as developing countries, estimated to occur in $2-4 \%$ of the adult population over 65 years. Percutaneous aortic valvular intervention has evolved for the treatment of aortic valve disease in elderly patients and ongoing trials will define the clinical role of this new therapy. ${ }^{10}$

So it is the high time to undertake active research to gain a better understanding of the disease in the elderly and provide effective managgement.

\section{References:}

1. Prakash R, Chowdhury SK, Singh US: A study of morbidity pattern among geriatric population in an urban area of Udaipur Rajasthan. Indian Journal of Community Medicine 2004; XXIX: 35-40.

2. Why Should I Be Interested in Learning About Older People? American Medical Student Association: 2008
3. Why Should I Consider Becoming a Geriatrician? American Medical Student Association: 2008

4. Fu M: Heart failure therapy in the elderly: Where are we? What are we doing? International Journal of Cardiology 2007 [Editorial].

5. Polena S, Mamakos E, Hague A et. al.: Secondary Pulmonary Hypertension In Geriatric Population. Chest 2005. 29: 469-475.

6. Sander GE: High Blood Pressure in the Geriatric Population: Treatment Considerations. The American Journal of Geriatric Cardiology. 2002; 11: 223232.

7. Alexander KP,Newby LK, Cannon CP et. al.American Heart Association Council on Clinical Cardiology; Society of Geriatric Cardiology. Acute coronary care in the elderly, part I: non ST-segment-elevation acute coronary syndrome: a scientific statement for healthcare professionals from the American Heart Association Council on Clinical Cardiology: in collaboration with the Society of Geriatric Cardiology. Circulation 2007; 115: 2549-2569.

8. Vrints CJM: Acute coronary syndrome in elderly. Eur. Heart $J$ 2008; 29: 1213-1214. [Editorial]

9. Devlin G, Gore JM, Elliot $\mathrm{J}$ et al. Management and 6 month outcomes in elderly and very elderly patients with high risk non-ST-elevation acute coronary syndromes: The Global Registry of Acute Coronary Events. Eur. Heart J 2008; 29: 1275-1282.

10. Cribier A, Eltchaninoff H, Tron C, Bauer F \& Gerber L: Percutaneous Implantation of Aortic Valve Prosthesis in Patients with Calcific Aortic Stenosis: Technical Advances, Clinical Results and Future Strategies Journal of Interventional Cardiology 2006; 19: S87S96. 